\title{
Averaged changes in the orbital elements of meteoroids due to Yarkovsky-Radzievskij force
}

\author{
Galina O. Ryabova ${ }^{1}$ \\ ${ }^{1}$ Tomsk State University, 634050 Tomsk, Russian Federation \\ email: ryabova@niipmm.tsu.ru
}

\begin{abstract}
Yarkovsky-Radzievskij effect exceeds the Poynting-Robertson effect in the perturbing action on particles larger than $100 \mu \mathrm{m}$. We obtained formulae for averaged changes in a meteoroid's Keplerian orbital elements and used them to estimate dispersion in the Geminid meteoroid stream. It was found that dispersion in semi-major axis of the model shower increased nearly three times on condition that meteoroids rotation is fast, and the rotation axis is stable.
\end{abstract}

Keywords. meteoroids, methods: analytical, celestial mechanics

To obtain the averaged changes in a meteoroid's orbit we use equations for the derivatives of the orbital elements in the form suggested by Burns (1976), namely $d b / d t=$ $F\left(a, \ldots, \omega, v, E, m, r, F_{r}, F_{t}, F_{n}\right)$, where $b$ is one of the standard Keplerian orbital elements $a, e, i, \Omega, \omega ; r$ is the heliocentric distance, $m$ is the meteoroid mass, $v$ is the true anomaly, $E$ is the eccentric anomaly, and $F_{r}, F_{t}, F_{n}$ are the radial, transverse, and normal components of the perturbing force. As in (Burns et al. 1979), we average the time variation of the elements over the orbital period under the assumption that $a$ and $e$ are essentially constant over this time interval and that the angular momentum is conserved. The average change in any element $b$ is then:

$$
\left\langle\frac{d a}{d t}\right\rangle=\frac{1}{P H} \oint \frac{d a}{d t} r^{2} d v,
$$

where $P$ is the orbital period and $H=\left[a \mu\left(1-e^{2}\right)\right]^{1 / 2}$ is the orbital angular momentum of the meteoroid per unit mass, $\mu$ is the gravitational constant of the sun.

The perturbing Yarkovsky-Radzievskij force for fast rotators, according to Burns et al. (1979) is

$$
F_{Y}=k_{Y} r^{-7 / 2}, \quad k_{Y}=2.962 r_{m}^{2} c^{-1} \sigma^{1 / 4}(1-\alpha)^{1 / 4} \gamma \omega_{m}^{-1 / 2} S_{0}^{7 / 4} r_{0}^{7 / 2} \cos \xi,
$$

where $(1 / \gamma)=300 \mathrm{~J} /\left(\mathrm{m}^{2} \cdot \mathrm{s}^{1 / 2} \cdot \mathrm{K}\right)$ is the thermal inertia, $r_{m}$ is the radius of the meteoroid, $\alpha=0.1$ is the albedo, $\omega_{m}=10^{4} \mathrm{rad} / \mathrm{s}$ is the angular velocity of the meteoroid $\left(r_{m}=\right.$ $1 \mathrm{~mm}), S_{0}$ is the solar constant at heliocentric distance $r_{0}=1 \mathrm{au}, \xi$ is the angle between the rotational axis of the particle and its orbital plane $\left(\xi=45^{\circ}\right.$ for prograde, or $\xi=135^{\circ}$ for retrograde rotation), $c$ is the speed of light, and $\sigma$ is the Stefan-Boltzmann constant.

The values of the parameters in (2) correspond to the model accepted by Olsson-Steel (1987). As in (Olsson-Steel 1987), we assume that $F_{r}=F_{t}=F_{n}=F_{Y} / \sqrt{3}$ due to the precession of the rotation axis and the frequent changes in the rotation speed. Even now, when computers are much faster, the numerical integration of (1) is still very expensive, so analytic expressions for the averaged changes in the orbital elements were found:

$$
\begin{array}{ll}
<\dot{a}>=k_{1} I_{52}, & <\dot{e}>=k_{2}\left(I_{32 c}+e I_{12}+I_{12 c}\right), \quad<i>=k_{2} I_{12 c} \cos \omega, \\
<\dot{\Omega}>=k_{2} I_{12 c} \sin \omega / \sin i, & <\dot{\omega}>=-k_{2} I_{32 c} / e-\cos i<\dot{\Omega}>,
\end{array}
$$


Table 1. Coefficients of expansion (3)

\begin{tabular}{|l|ccccccccc|}
\hline Integrals & $p_{0}$ & $p_{1}$ & $p_{2}$ & $p_{3}$ & $p_{4}$ & $p_{5}$ & $p_{6}$ & $p_{7}$ & $p_{8} \mid$ \\
\hline$I_{52}$ & 2 & 0 & $\frac{15}{8}$ & 0 & $-\frac{15}{2 \cdot 16^{2}}$ & 0 & $-\frac{25}{2 \cdot 16^{3}}$ & 0 & $-\frac{1575}{2 \cdot 16^{5}}$ \\
\hline$I_{32 c}$ & 0 & $\frac{3}{2}$ & 0 & $-\frac{3}{4 \cdot 16}$ & 0 & $-\frac{15}{2 \cdot 16^{2}}$ & 0 & $-\frac{315}{2 \cdot 16^{4}}$ & 0 \\
\hline$I_{12}$ & 2 & 0 & $-\frac{1}{8}$ & 0 & $-\frac{15}{2 \cdot 16^{2}}$ & 0 & $-\frac{105}{2 \cdot 16^{3}}$ & 0 & $-\frac{15015}{2 \cdot 16^{5}}$ \\
\hline$I_{12 c}$ & 0 & $\frac{1}{2}$ & 0 & $\frac{3}{4 \cdot 16}$ & 0 & $\frac{35}{8 \cdot 16^{2}}$ & 0 & $-\frac{1155}{2 \cdot 16^{4}}$ & 0 \\
\hline
\end{tabular}

where

$$
\begin{array}{ll}
k_{1}=k_{Y} /\left[\pi \sqrt{3 \mu} m a^{2}\left(1-e^{2}\right)^{5 / 2}\right], & k_{2}=k_{Y} /\left[2 \pi \sqrt{3 \mu} m a^{3}\left(1-e^{2}\right)^{3 / 2}\right], \\
I_{52}=\oint(1+e \cos v)^{5 / 2} d v, & I_{32 c}=\oint(1+e \cos v)^{3 / 2} \cos v d v, \\
I_{12}=\oint(1+e \cos v)^{1 / 2} d v, & I_{12 c}=\oint(1+e \cos v)^{1 / 2} \cos v d v .
\end{array}
$$

The integrals $I$ are easily found as series by expanding the integrands using the binomial formula

$$
I=\pi \sum_{j=0}^{\infty} p_{j} e^{j} .
$$

Accuracy to $1 \%$ is achieved by keeping terms to eighth order in the eccentricity. The expansion coefficients $p_{j}$ in (3) are given in Table 1.

Using the formulae we made some estimations for the Geminid meteoroid stream. The model of the stream was like in (Ryabova 2007) and the meteoroid mass was taken as $3 \times 10^{-3} \mathrm{~g}$. Direction of rotation (prograde or retrograde) for each meteoroid was chosen using a pseudorandom number generator. The dispersion of the Geminids is anisotropic, so the total dispersion and the dispersion observed at the Earth differ. The width of the model shower is about $1.5-2.5^{\circ}$ in solar longitude (Ryabova 2007). With YR-addition the width increases by $0.3^{\circ}$, remaining less than the observed width $5^{\circ}$ (Fox et al. 1983). As to the semi-major axis, YR-effect increases $\Delta a$ for the model shower nearly 3 times. We found that YR-effect, being the mass-dependant, increases the mass separation in the stream about twice.

\section{Acknowledgement}

The author acknowledges the financial support from IAU. Some part of funding was provided by Tomsk State University Competitiveness Improvement Program. The author is grateful to the anonymous reviewer. $\dagger$

\section{References}

Burns, J. A. 1979, Am. J. Phys., 44, 944

Burns, J. A., Lamy, P. L., \& Soter, S. 1979, Icarus, 40, 1

Fox, K., Williams, I. P. \& Hughes, D. W. 1983, MNRAS, 205, 1155

Olsson-Steel, D. 1987, MNRAS, 226, 1

Ryabova, G. O. 1990, Sol. Syst. Res., 24, 169

Ryabova, G. O. 2007, MNRAS, 375, 1371

$\dagger$ Part of this research was firstly fulfilled about 25 years ago. It remained unnoticed to meteor astronomers community, because the English translation (Ryabova 1990) of the paper was published in a hard-to-reach journal and, until recently, was absent from SAO/NASA ADS database. The method was carefully revised and some corrections were introduced. 